\title{
Tomato 14-3-3 Proteins Are Required for Xv3 Disease Resistance and Interact with a Subset of Xanthomonas euvesicatoria Effectors
}

\author{
Zoe Dubrow, ${ }^{1}$ Sukumaran Sunitha, ${ }^{2}$ Jung-Gun Kim, ${ }^{1}$ Chris D. Aakre, ${ }^{1}$ Anil Madhusoodana Girija, ${ }^{2}$ \\ Guy Sobol, ${ }^{2}$ Doron Teper, ${ }^{2}$ Yun Chu Chen, ${ }^{1}$ Nejla Ozbaki-Yagan, ${ }^{1}$ Hillary Vance, ${ }^{1}$ Guido Sessa, ${ }^{2}$ and \\ Mary Beth Mudgett ${ }^{1,+}$ \\ ${ }^{1}$ Department of Biology, Stanford University, Stanford, CA 94305-5020, U.S.A.; and ${ }^{2}$ School of Plant Sciences and Food \\ Security, Tel Aviv University, 69978 Tel Aviv, Israel
}

Accepted 25 June 2018.

The 14-3-3 phospho-binding proteins with scaffolding activity play central roles in the regulation of enzymes and signaling complexes in eukaryotes. In plants, 14-3-3 isoforms are required for disease resistance and key targets of pathogen effectors. Here, we examined the requirement of the tomato (Solanum lycopersicum) 14-3-3 isoform (TFT) protein family for $X v 3$ disease resistance in response to the bacterial pathogen Xanthomonas euvesicatoria. In addition, we determined whether TFT proteins interact with the repertoire of $X$. euvesicatoria type III secretion effector proteins, including AvrXv3, the elicitor of $X v 3$ resistance. We show that multiple TFT contribute to $X v 3$ resistance. We also show that one or more TFT proteins physically interact with multiple effectors (AvrXv3, XopE1, XopE2, XopN, XopO, XopQ, and XopAU). Genetic analyses indicate that none of the identified effectors interfere with AvrXv3-elicited resistance into $X v 3$ tomato leaves; however, XopE1, XopE2, and XopO are required to suppress symptom development in susceptible tomato leaves. Phospho-peptide mapping revealed that XopE2 is phosphorylated at multiple residues in planta and residues T66, T131, and $\mathrm{S334}$ are required for maximal binding to TFT10. Together, our data support the hypothesis that multiple TFT proteins are involved in immune signaling during $X$. euvesicatoria infection.

The 14-3-3 proteins are a class of phospho-binding proteins that participate in numerous signaling pathways in all eukaryotes (Fu et al. 2000). The 14-3-3 proteins often bind protein substrates (i.e., "client proteins") at one of three phosphorylation-dependent binding motifs (i.e., mode 1: RSXpSXP, mode 2: RXY/FXpSXP, or mode 3: pS/pTXXCOOH), where key serine (S) or threonine (T) residues are phosphorylated (i.e., $\mathrm{pS}$ or $\mathrm{pT}$, respectively)

Zoe Dubrow and Sukumaran Sunitha are co-first authors.

${ }^{\dagger}$ Corresponding author: Mary Beth Mudgett;

E-mail: mudgett@stanford.edu

Funding: This work was supported by the United States-Israel Binational Science Foundation (grants 2011069 and 2015062 to G. Sessa and M. B. Mudgett) and National Science Foundation (grant IOS-1555957 to M. B. Mudgett).

*The $\boldsymbol{e}$-Xtra logo stands for "electronic extra" and indicates that nine supplementary figures and three supplementary tables are published online.

(c) 2018 The American Phytopathological Society
(Coblitz et al. 2005; Ganguly et al. 2005; Madeira et al. 2015; Muslin et al. 1996; Yaffe et al. 1997), although phosphorylation is not always required (Borch et al. 2002; Petosa et al. 1998). The 14-3-3 proteins function as protein scaffolds influencing the stability or activity of their client proteins (Bridges and Moorhead 2005). For example, 14-3-3 proteins can form dimers to facilitate protein-protein interaction between two clients, alter the subcellular localization of a client, protect clients from posttranslational modifications (e.g., phosphorylation, dephosphorylation, or ubiquitination), and change client enzymatic activity. These functions enable cells to ultimately control the kinetics and magnitude of signal transduction pathways in response to diverse stimuli.

The 14-3-3 proteins play central roles in immune signaling pathways in both plants and animals. In plants, 14-3-3 proteins comprise large gene families and various isoforms have been linked to proper execution of disease resistance pathways associated with pattern-triggered immunity (PTI) and effectortriggered immunity (ETI) (Bigeard et al. 2015; Cui et al. 2015). During PTI, pattern-recognition receptors detect the presence of microbe-associated molecular patterns (MAMP; e.g., bacterial flagellin and fungal chitin) and damage-associated molecular patterns (DAMP; e.g., oligogalacturonides and elicitor peptides) and activate downstream defense signaling (Choi and Klessig 2016). Notably, recent work indicates that the human 14-3-3 isoform eta is a DAMP linked with rheumatoid arthritis (Maksymowych et al. 2014), suggesting that 14-3-3 proteins may also serve as immune elicitors following pathogen invasion in animal cells. During ETI, intracellular nucleotide-binding and leucine-rich repeat immune receptors known as nucleotidebinding leucine-rich repeat resistance proteins detect pathogen effectors delivered inside plant cells and activate robust defense responses leading to local and systemic immunity.

Although 14-3-3 proteins have been linked to plant defense, little is known about the diversity of their clients or how specific 14-3-3 isoforms regulate defense signal transduction for a given plant species. What is known is that transcription of various plant 14-3-3 proteins is highly upregulated in response to infection (Roberts and Bowles 1999), and distinct isoforms interact with core defense components required for PTI or ETI (Lozano-Durán and Robatzek 2015). For example, three Arabidopsis 14-3-3 isoforms (GF-14- $\omega$, GF-14- $\phi$, and GF-14$\psi)$ stimulate the activity of the calcium-dependent protein kinase CPK-1 (Camoni et al. 1998), whereas five (GF-14- $\omega$, GF-14-ı, GF-14-o, GF-14-א, and GF-14- $\phi$ ) interact with multiple 1-aminocyclopropane-1-carboxylic acid synthase (ACS) isoforms, impacting their stability and ethylene biosynthesis 
(Yoon and Kieber 2013). In addition, Arabidopsis GF-14- $\gamma$ binds the atypical resistance protein RPW8.2 and is implicated in the regulation of programmed cell death (PCD) and resistance to powdery mildew (Yang et al. 2009). In tomato, 14-33 isoform 7 (TFT7) interacts with mitogen-activated protein (MAP) kinases MAPKKK $\alpha$ and MKK2, two positive regulators of ETI-associated PCD controlling resistance to Pseudomonas syringae (Oh and Martin 2011; Oh et al. 2010).

The 14-3-3 proteins also interact with pathogen effector proteins from animal and plant pathogens (Fu et al. 2000; Lozano-Durán and Robatzek 2015). This suggests that pathogens may target 14-3-3 isoforms to modulate the activity of their effectors inside eukaryotic host cells or interfere with 143-3-regulated signaling pathways that control the outcome of host-pathogen interactions. Evidence supporting both scenarios exists. For example, the binding of the exoenzyme $\mathrm{S}$ (ExoS), a type III secreted effector (T3SE), from $P$. aeruginosa to human 14-3-3 isoforms is required to activate ExoS-dependent ADP-ribosyltransferase activity (Henriksson et al. 2002) and cell death in the mouse model for pneumonia (Ottmann et al. 2007). By contrast, multiple T3SE from plant-pathogenic bacteria (e.g., XopN, XopQ, and AvrRxv from Xanthomonas euvesicatoria and HopQ and HopM1 from P. syringae) antagonize 14-3-3 defense signaling. XopN and HopM1 suppress PTI (Lozano-Durán et al. 2014; Nomura et al. 2006; Taylor et al. 2012), whereas XopQ and HopQ suppress ETI (Li et al. 2013; Teper et al. 2014). AvrRxv and XopN appear to sequester TFT proteins (TFT9 and TFT1, respectively) in order to promote virulence (Taylor et al. 2012; Whalen et al. 2008), whereas HopM1 suppresses MAMP-triggered reactive oxygen species production and stomatal immunity (Lozano-Durán et al. 2014). Phosphorylation of XopN, XopQ, and HopQ is required for binding to 14-3-3 proteins (Giska et al. 2013; Li et al. 2013; Taylor et al. 2012; Teper et al. 2014), suggesting that phosphorylation of pathogen T3SE inside plant host cells may be critical for complex formation.

To gain further insight into the extent to which 14-3-3 proteins participate in immune signaling and are targets of pathogen effectors, we investigated the contribution of the 14-3-3 gene family in $X v 3$ disease resistance in tomato during $X$. euvesicatoria infection. The TFT family consists of 11 isoforms, TFT1 to TFT11 (Roberts and Bowles 1999). We also determined whether TFT proteins interact with AvrXv3 or the T3SE from the well-characterized strain $X$. euvesicatoria $85-10$ and characterized the biochemical features impacting 14-3-3effector complex formation. Our data reveal that multiple TFT contribute to $X v 3$-mediated immunity. Moreover, we demonstrate that AvrXv3 and six of $38 X$. euvesicatoria T3SE examined physically interact with $14-3-3$ proteins in planta. These include the novel 14-3-3-interacting effectors XopE1, XopE2, $\mathrm{XopO}$, and XopAU, as well as XopN and XopQ, two known 143-3-interacting effectors (Kim et al. 2009; Taylor et al. 2012; Teper et al. 2014). We also provide evidence that XopE1, XopE2, and XopO are required for $X$. euvesicatoria symptom development in susceptible tomato leaves. Collectively, our findings indicate that multiple TFT proteins are required for immune signaling during $X$. euvesicatoria infection and may be susceptibility factors for a suite of $X$. euvesicatoria T3SE.

\section{RESULTS}

\section{Abundance of TFT mRNAs increases during $X$. euvesicatoria infection.}

To understand which 14-3-3 proteins are involved in $X v 3$ mediated disease resistance in tomato, we quantified TFT mRNA abundance during compatible and incompatible $X$. euvesicatoria-tomato interactions using $X$. euvesicatoria strain 5746 and Hawaii 7981 tomato plants. Hawaii 7981 tomato plants carrying the $X v 3$ resistance gene are susceptible to $X$. euvesicatoria strain 5746 (compatible interaction) and are resistant to $X$. euvesicatoria strain 5746 expressing avrXv3 (incompatible interaction or ETI) (Astua-Monge et al. 2000). mRNA abundance of the MAP kinase $M P K 3$ was used as a positive control as it accumulates during ETI (Balaji et al. 2007). Compared with mock infiltrated leaves, the mRNA abundance of several TFT increased significantly at $24 \mathrm{~h}$ postinoculation (hpi) in response to both infections (Fig. 1). The highest mRNA levels were detected during avrXv3-triggered ETI for TFT1, TFT4, TFT5, TFT6, TFT10, and TFT11.

\section{Multiple TFT proteins contribute to ETI.}

Next, we used virus-induced gene silencing (VIGS) to determine whether TFT, whose mRNA accumulated during avrXv3-triggered ETI (TFT1, TFT4, TFT5, TFT6, TFT10, and TFT11), play a major role in anti-X. euvesicatoria immunity. The Tobacco rattle virus (TRV)-based system was used to independently silence each of the selected TFT in Hawaii $7981 X v 3$ plants. For each silenced tomato line, mRNA levels for all TFT were measured by quantitative polymerase chain reaction (qPCR) to determine the specificity and magnitude of gene silencing (Supplementary Fig. S1). In general, each VIGS construct preferentially reduced expression of the target TFT gene by 40 to $60 \%$ (with the exception of TFT5, whose expression was reduced by $20 \%$ ). Minor off-target silencing was detected in plants silenced with TFT5, TFT10, and TFT11 constructs, possibly due to sequence similarity between isoforms. Of the constructs tested, those targeting TFT4 and TFT6 appeared to be specific.

Bacterial growth curves were then performed for each TFTsilenced line along with an empty vector control (TRV2). Leaves were inoculated with a $1 \times 10^{5} \mathrm{CFU} / \mathrm{ml}$ suspension of $X$. euvesicatoria 5746 expressing avrXv3. Bacterial titer was quantified at 0,4 , and 8 days postinoculation (dpi). At 8 dpi, the six TFT-silenced lines (i.e., TFT1, TFT4, TFT5, TFT6, TFT10, and TFT11) tended to have higher titers of $X$. euvesicatoria compared with the TRV2 vector control (Fig. 2A). However, a statistically significant $(P$ value $<0.05)$ increase in $X$. euvesicatoria titer was only detected in TFT4, TFT6, and TFT10-silenced leaves. The higher $X$. euvesicatoria titers detected in all six of the TFT-silenced lines correlated with increased symptom development at 8 dpi. Compared with the empty vector control, the TFT-silenced leaves had more bacterial spot lesions, yellowing of tissue (chlorosis), and tissue damage (necrosis) (Fig. 2B).

In terms of electrolyte leakage detected during the hypersensitive response, all six $T F T$-silenced lines tended to release fewer electrolytes from the $X$. euvesicatoria avrXv3-inoculated leaves at 24 and 36 hpi (Fig. 2C). However, a significant decrease $(P$ value $<0.05)$ in electrolyte leakage from infected leaves was only observed for TFT1, TFT4, TFT5, TFT6, and TFT10 (Fig. 2C). Taken together, these data indicate that multiple isoforms contribute to $X v 3$-mediated resistance in tomato in response to $X$. euvesicatoria expressing avrXv3.

\section{AvrXv3 and multiple $X$. euvesicatoria 85-10 effectors interact with TFT in yeast.}

Prior work has shown that individual effectors from a particular pathogen interact with one or more 14-3-3 isoforms (Fu et al. 2000; Lozano-Durán and Robatzek 2015). Given that three effectors (AvrRxv, XopN, and XopQ) from X. euvesicatoria were found to interact with multiple TFT proteins (Kim et al. 2009; Teper et al. 2014; Whalen et al. 2008), we hypothesized that $X$. euvesicatoria and potentially other phytopathogenic bacteria have evolved multiple T3SE to target one or more 14-3-3 
isoforms to directly alter plant immune signaling or metabolism during infection. To begin to test this hypothesis, we used three prediction programs (ANN, PSSM, and SVM) (Madeira et al. 2015) to identify putative 14-3-3 phospho-binding sites in AvrXv3 (Astua-Monge et al. 2000) T3SE from X. euvesicatoria strain $85-10$. We chose $X$. euvesicatoria strain $85-10$ because the genome sequence is available and the effector repertoire is well characterized (Potnis et al. 2011; Roden et al. 2004; Schulze et al. 2012; Teper et al. 2016; Thieme et al. 2005).

Of 39 T3SE examined, most are predicted to have two or more 14-3-3 phospho-binding sites (Supplementary Table S1). To determine the extent to which Xanthomonas T3SE bind 14-3-3 proteins, we performed a targeted LexA-based protein interaction screen in yeast with AvrXv3 or 38 $X$. euvesicatoria T3SE and 11 TFT (TFT1 to TFT11). Five effectors autoactivated the system (XopD, XopE2, XopI, XopS, and XopAW), preventing further analysis in yeast. Of the remaining 34 effectors, 9 (AvrXv3, AvrBs2, XopE1, XopN, XopO, XopP, XopQ, XopZ1, and XopAU) interacted with multiple TFT to varying degrees (Supplementary Fig. S2). XopN and XopQ interaction data were not included here because they were previously reported (Kim et al. 2009; Teper et al. 2014).

Bimolecular fluorescence complementation analysis shows that XopE2 and XopAU interact with 14-3-3 proteins in planta.

Bimolecular fluorescence complementation (BiFC) assays were performed in Nicotiana benthamiana to establish whether any of the new effector-TFT complexes detected in yeast form in plant cells. Seven $X$. euvesicatoria effectors
(XopE1, XopE2, XopO, XopP, XopZ1, AvrBs2, and XopAU) and a $\beta$-glucuronidase (GUS) control were each cloned into pDEST- ${ }^{-{ }^{2}}$ SCYCE to create effector-HA-SCFP3A ${ }^{\mathrm{C}}$ and GUS-HA-SCFP3 $\mathrm{A}^{\mathrm{c}}$ fusion proteins, respectively. XopE2, which could not be tested in yeast due to autoactivation, was included in this analysis because it shares high similarity with XopE1 (Thieme et al. 2007), one of the effectors identified in our yeast screen. TFT10 and GUS were cloned into pDEST- ${ }^{G W}$ VYNE to create TFT10-cMyc-VENUS ${ }^{\mathrm{N}}$ and GUScMyc-VENUS $^{\mathrm{N}}$ fusion proteins, respectively. We used TFT10 for BiFC because most of the effectors strongly interacted with this isoform in yeast and it contributes significantly to anti$X$. euvesicatoria defense (Figs. 1 and 2). The XopN/TFT1 pair was used as a positive control because a physical interaction between these proteins has been previously demonstrated by BiFC and affinity purification (Taylor et al. 2012). GUS was used in GUS/TFT and effector/GUS pairs as a negative control (Taylor et al. 2012). Leaves were inoculated with two Agrobacterium tumefaciens strains expressing the BiFC fusion proteins and then analyzed by confocal microscopy at $48 \mathrm{hpi}$ (Fig. 3) and immunoblot analysis (Supplementary Fig. S3).

Bright fluorescence was observed near the plasma membrane of the epidermal cells expressing XopE2 and TFT10 (Fig. 3D), similar to that for XopN/TFT1 (Fig. 3A). This was expected because both XopE2 and XopN are known to localize to the plasma membrane of epidermal cells (Taylor et al. 2012; Thieme et al. 2007). By contrast, weak fluorescence was observed for the two negative control pairs, GUS/TFT10 (Fig. 3B) and XopE2/GUS (Fig. 3C). XopAU interaction with TFT could not be examined using this system because XopAU elicits cell death in $N$. benthamiana (Teper et al. 2018). Using a kinase-deficient

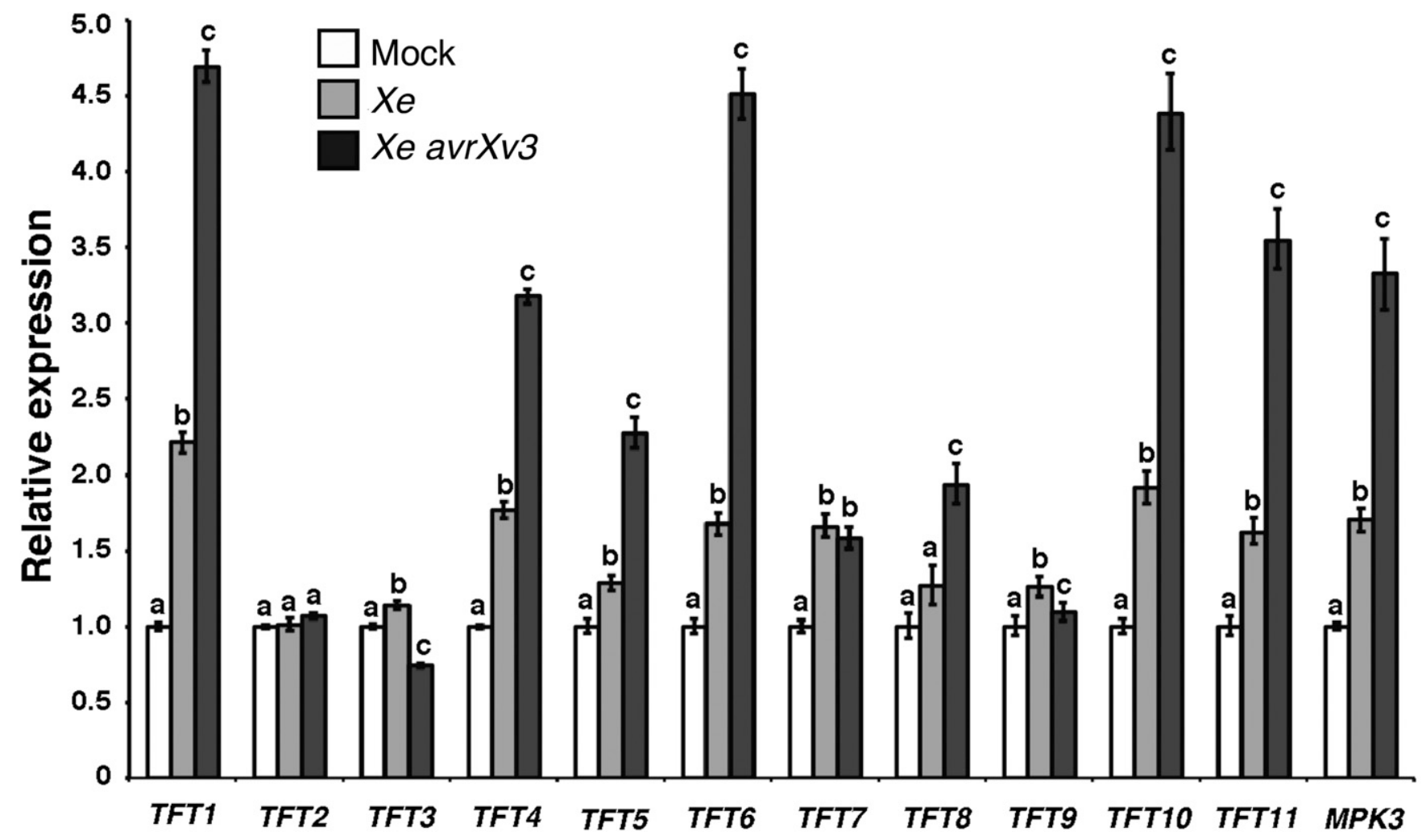

Fig. 1. Tomato 14-3-3 isoform (TFT) mRNA abundance in Hawaii 7981 tomato $X v 3$ leaves during compatible and incompatible interactions. Leaves were vacuum infiltrated with a $10 \mathrm{mM} \mathrm{MgCl}_{2}$ solution (white bars; mock) or with a bacterial suspension $\left(1 \times 10^{8} \mathrm{CFU} / \mathrm{ml}\right)$ of Xanthomonas euvesicatoria $($ Xe $)$ strain 5746 (gray bars; compatible) or X. euvesicatoria 5746 avrXv3 (black bars; incompatible). TFT1 to TFT11 and MPK3 mRNA levels were quantified by quantitative polymerase chain reaction at $24 \mathrm{~h}$ postinoculation. Relative expression (mean \pm standard error, $n=5$ ) was determined against the mean of mockinoculated samples. Letters denote significant differences based on analysis of variance and comparisons for all pairs using the Tukey-Kramer honestly significant difference test $(P$ value $<0.05)$. The experiment was repeated three times with similar results. 


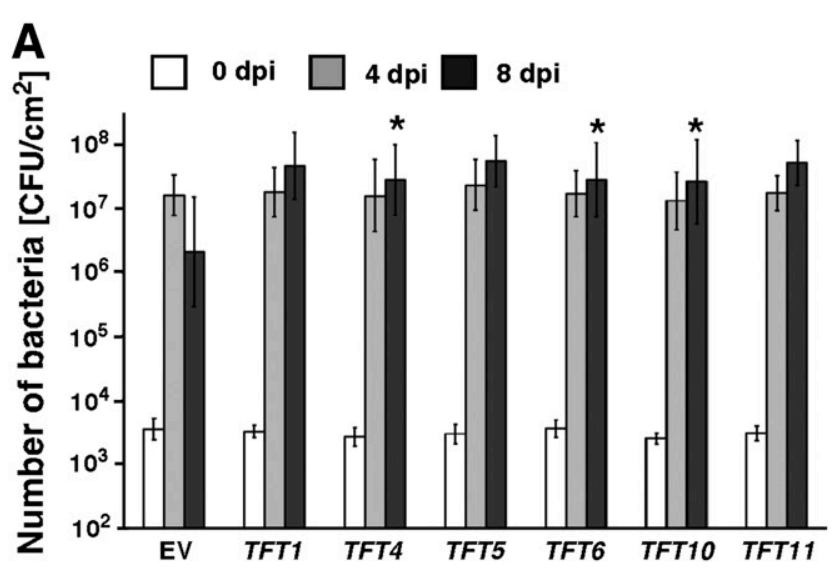

\section{B}
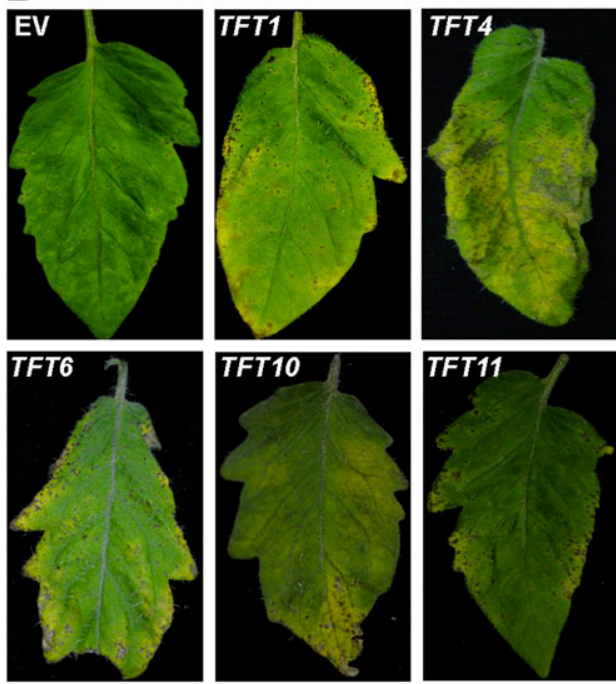

C

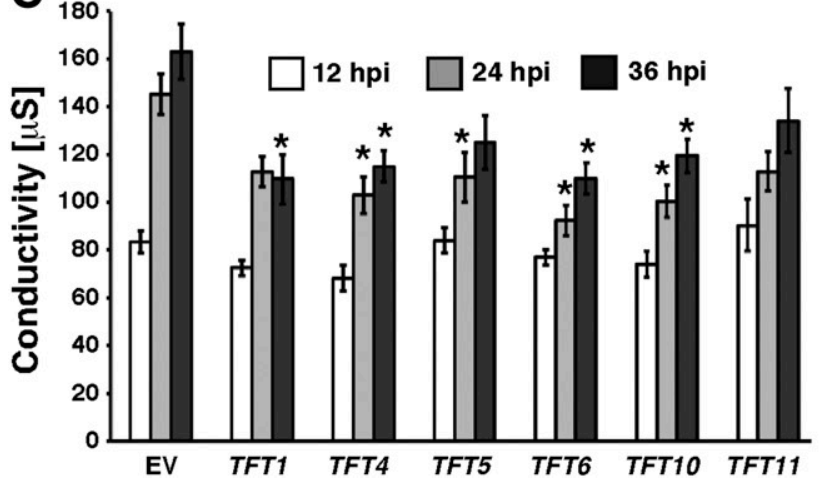

Fig. 2. Virus-induced gene silencing of individual tomato 14-3-3 isoforms (TFT) in resistant Hawaii $7981 X v 3$ plants inhibits effector-triggered immunity triggered by Xanthomonas euvesicatoria 5746 expressing avrXv3. A, Number of bacteria in tomato plants infected with a Tobacco rattle virus 2 vector either empty $(\mathrm{EV})$ or carrying gene fragments for silencing of the indicated TFT $(1,4,5,6,10$, or 11$)$. Leaves were vacuum infiltrated with a bacterial suspension of $1 \times 10^{5} \mathrm{CFU} / \mathrm{ml}$ and the number of bacteria was determined at 0,4 , and 8 days postinoculation (dpi). Data are mean $\mathrm{CFU} / \mathrm{cm}^{2} \pm$ standard error (SE) $(n=3$ biological repeats). Asterisks denote significant differences in all three biological repeats based on analysis of variance and comparisons for all pairs (honestly significant difference [HSD]; $P$ value $<0.05)$. B, Disease symptoms of leaves infected in A at 8 dpi. C, Quantification of cell death in tomato plants silenced as in A and vacuum infiltrated with a bacterial suspension of $1 \times 10^{8} \mathrm{CFU} / \mathrm{ml}$. Electrolyte leakage was measured at 12,24 , and $36 \mathrm{~h}$ postinoculation (hpi). Data are mean conductivity $\pm \mathrm{SE}(n=7)$. Asterisks denote significant differences based on analysis of variance and comparisons for all pairs (HSD; $P$ value $<$ $0.05)$. The experiment was repeated three times with similar trends. variant of XopAU (XopAU $\mathrm{K}_{\mathrm{K} 240 \mathrm{~A}}$ ), which does not cause cell death in $N$. benthamiana leaves, and a split-luciferase complementation assay, we were able to detect interaction of XopAU $U_{\mathrm{K} 240 \mathrm{~A}}$ with TFT1 and TFT7 (Supplementary Fig. S4), indicating that XopAU interacts in planta with at least two 14-3-3 isoforms.

Notably, fluorescence was not observed when TFT10 was coexpressed with AvrBs2 (Fig. 3E). Immunoblot analysis revealed that the AvrBs2 fusion protein was expressed, suggesting that AvrBs2 does not interact with TFT10 in planta. The weak AvrBs2-TFT isoform interactions observed in yeast may be artifacts (false positives). No conclusions could be made for XopE1, XopO, and XopZ1 because we were unable to detect protein expression by immunoblot analysis.

Affinity purification of XopE1, XopE2, XopO, and AvrXv3 with 14-3-3 proteins.

We used $\mathrm{Ni}^{2+}$-NTA affinity purification to monitor effector-TFT complexes in N. benthamiana leaf extracts. Given that effector expression was problematic using the BiFC constructs, we attempted to purify His-tagged effectors (XopE1-His, XopE2-His, XopO-His, XopP-His, XopZ1-His, and AvrBs2-His) that were transiently coexpressed with TFT5-cMyc-VENUS ${ }^{\mathrm{N}}$ or TFT10-cMyc-VENUS ${ }^{\mathrm{N}}$. We were able to purify high levels of XopE2-His and AvrBs2-His and low levels of XopE1-His and XopO-His. XopP-His and XopZ1-His were not enriched by affinity purification and could not be further assessed. XopE1-His, XopE2-His, and XopO-His were coexpressed with TFT10cMyc-VENUS $^{\mathrm{N}}$. Affinity purification revealed that all three effectors copurify with TFT10 (Fig. 4A). AvrBs2 was coexpressed with TFT5-cMyc-VENUS ${ }^{\mathrm{N}}$ (the isoform that interacted most strongly with AvrBs2 in yeast). AvrBs2 did not copurify with TFT5 (Fig. 4A), further suggesting that AvrBs2 does not interact with TFT in planta. In subsequent tests, we coexpressed AvrXv3His with TFT10-GFP and were able to copurify AvrXv3 with TFT10 (Fig. 4B). Collectively, these effector-TFT interaction studies in $N$. benthamiana demonstrate that XopE1, XopE2, XopO, and AvrXv3 physically interact with TFT10.

\section{Role of effectors in $X v 3$ resistance.}

We hypothesized that Xanthomonas effectors that interact with 14-3-3 proteins might be targeting a 14-3-3 immune complex that is required for $X v 3$ resistance in tomato. To test this, we examined resistance phenotypes elicited by eight $X$. euvesicatoria strain 85-10 effector null mutants expressing AvrXv3 in $X v 3$ tomato leaves. The strains included five existing mutants ( $\triangle x o p A U, \Delta x o p N, \Delta x o p O, \Delta x o p P$, and $\Delta x o p Q)$ (Roden et al. 2004; Teper et al. 2014, 2018) and three mutants created in this study ( $\triangle x o p E 1, \Delta x o p E 2$, and $\Delta x o p E 1 \Delta x o p E 2)$. Tomato $X v 3$ tomato leaves were inoculated with a high titer $\left(1 \times 10^{8}\right.$ $\mathrm{CFU} / \mathrm{ml}$ ) of wild-type or mutant $X$. euvesicatoria $85-10$ bacteria carrying AvrXv3; then, electrolyte leakage and bacterial growth were measured. In general, we measured higher conductivity for tomato $X v 3$ leaves infected with $X$. euvesicatoria $\Delta x o p A U+A v r X v 3, X$. euvesicatoria $\Delta x o p P+A v r X v 3$, or $X$. euvesicatoria $\Delta x o p E 1 \Delta x o p E 2+$ AvrXv3 compared with leaves infected with wild-type $X$. euvesicatoria + AvrXv3 (Supplementary Fig. S6). However, bacterial titer for these mutants in infected tomato leaves was similar to that of wildtype $X$. euvesicatoria. These data suggest that the $X$. euvesicatoria 85-10 effectors found to interact with 14-3-3 proteins are not interfering with $X v 3$ resistance in tomato.

\section{XopE1, XopE2, and XopO suppress}

$X$. euvesicatoria-induced chlorosis in tomato leaves.

Next, we determined whether the $X$. euvesicatoria effector mutants were compromised in their ability to multiply within susceptible tomato leaves and cause disease symptoms. We 
analyzed four mutants $(\Delta x o p E 1, \Delta x o p E 2, \Delta x o p E 1 \Delta x o p E 2$, and $\Delta x o p O)$ on susceptible VF36 tomato plants because $X$. euvesicatoria 85-10 causes more disease symptoms in VF36 leaves compared with that of Hawaii 7981 leaves, enabling us to detect potential virulence phenotypes among the mutant strains. The titer of the X. euvesicatoria wild-type and mutant strains was similar (Supplementary Fig. S7), even at early time points. However, leaves infected with $\triangle x o p E 1, \Delta x o p E 2$, or $\triangle x о p O$ developed more chlorosis (i.e., yellowing) compared with leaves infected with the wild type (Fig. 5). The chlorosis phenotype appeared to be additive for the $X$. euvesicatoria $\Delta x o p E 1 / \Delta x o p E 2$ double mutant (Fig. 5). We observed chlorosis and tissue collapse in leaves infected with $X$. euvesicatoria $\Delta x o p E 1 / \Delta x o p E 2$ earlier (typically 1 to 2 days) than that observed for leaves infected with the single deletion mutants. These data indicate that XopE1, XopE2, and XopO are required to suppress symptom development in X. euvesicatoria-infected VF36 tomato leaves.

XopE2 is phosphorylated in planta.

We selected two effectors (XopE2 and XopO) to investigate the relationship between effector phosphorylation in planta and
14-3-3 binding. XopE2 and XopO were selected because we were able to establish protein purification schemes sufficient for phosphate affinity sodium dodecyl sulfate polyacrylamide gel electrophoresis (SDS-PAGE) analysis (i.e., Phos-tag analysis) and mass spectrometry (MS) analysis. First, we determined whether XopE2 and XopO are phosphorylated in plant extracts. XopE2-His and XopO-His were transiently expressed in $N$. benthamiana leaves and then purified from leaf extracts under denaturing conditions using $\mathrm{Ni}^{2+}$-NTA affinity chromatography. Protein was incubated with or without calf intestinal alkaline phosphatase (CIAP) and then separated by Phos-tag SDS-PAGE. CIAP treatment altered XopE2-His mobility (Fig. 6A), suggesting that XopE2 is phosphorylated. By contrast, no obvious mobility shifts were observed for XopO-His.

The 14-3-3 proteins are known to bind client proteins at conserved recognition motifs that contain phosphorylated serine or threonine residues (Muslin et al. 1996; Yaffe et al. 1997). Putative 14-3-3 phospho-binding sites were identified in both XopE2 and XopO; however, none of the predicted sites perfectly matched any of the three known 14-3-3 motifs; that is, mode I: RSX(pS/pT)XP; mode 2; RX(F/Y)X $(\mathrm{pS}) \mathrm{XP}$; or mode 3: (pS/pT)XX-COOH (Coblitz et al. 2005; Ganguly et al. 2005;

\section{A XopN-HA-SCFP3AC + TFT1-CMyC-VENUS}

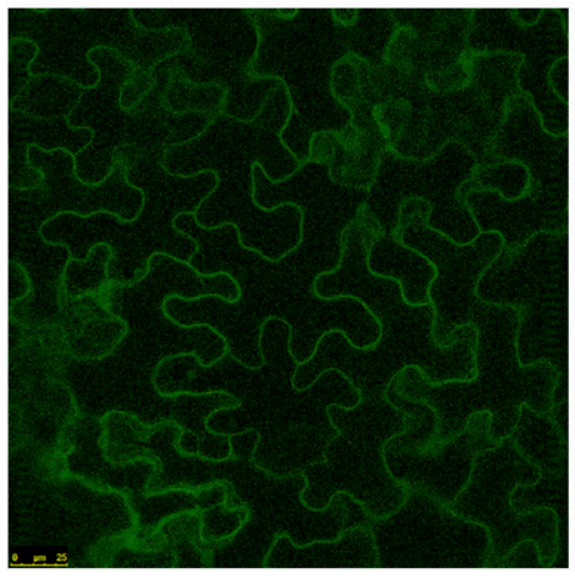

D
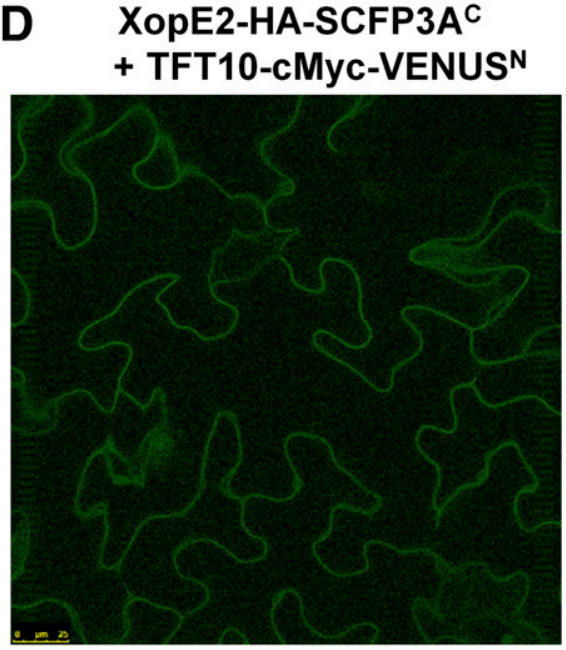

B GUS-HA-SCFP3AC + TFT10-cMyc-VENUS

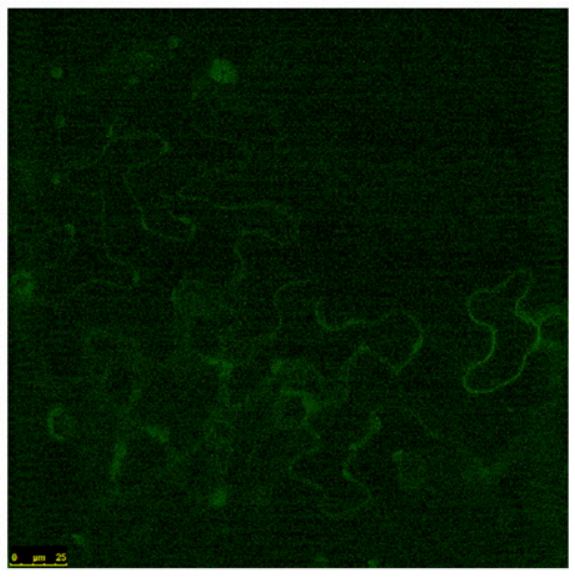

$\mathbf{E}$

\section{XopE2-HA-SCFP3AC + GUS-CMyC-VENUS ${ }^{\mathrm{N}}$}

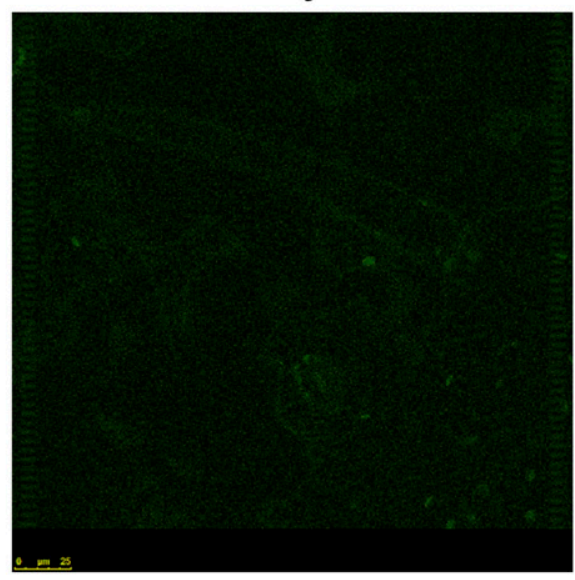

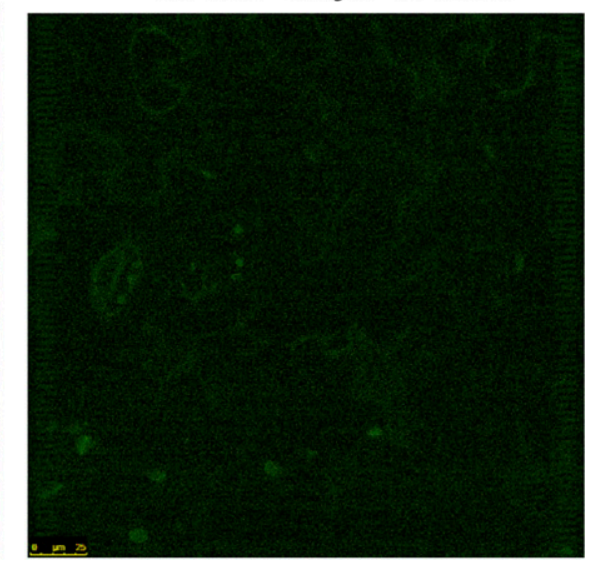

Fig. 3. Bimolecular fluorescence complementation analysis of XopE2 and AvrBs2 with tomato 14-3-3 isoform (TFT)10 in planta. Nicotiana benthamiana leaves inoculated with a suspension $\left(0.8 \times 10^{9} \mathrm{CFU} / \mathrm{ml}\right)$ of two Agrobacterium tumefaciens strains expressing different fusion proteins. Leaf epidermal cells

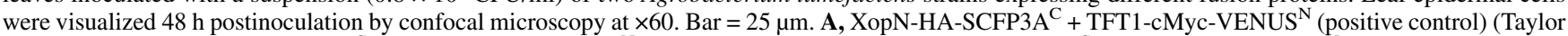
et al. 2012). B, GUS-HA-SCFP3A ${ }^{\mathrm{C}}+$ TFT10-cMyc-VENUS $^{\mathrm{N}}$ (negative control). C, XopE2-HA-SCFP3A ${ }^{\mathrm{C}}+\mathrm{GUS}^{\mathrm{N}-\mathrm{CMyc}-\mathrm{VENUS}}{ }^{\mathrm{N}}$ (negative control). D, XopE2-HA-SCFP3A ${ }^{\mathrm{C}}+$ TFT10-cMyc-VENUS $^{\mathrm{N}}$. E, AvrBs2-HA-SCFP3A ${ }^{\mathrm{C}}+$ TFT10-cMyc-VENUS $^{\mathrm{N}}$. 
Muslin et al. 1996; Yaffe et al. 1997). To map the phosphorylation sites in XopE2 and XopO, we digested purified XopE2His and XopO-His with trypsin, performed phospho-peptide enrichment, and then analyzed the peptides by liquid chromatography-MS analysis. This analysis revealed phosphorylated residues for both XopE2 and XopO (Fig. 6B).

For XopO, the peptides analyzed by MS covered $97.8 \%$ of the mature polypeptide. Only one phosphorylated residue, S47, was detected. This residue was only phosphorylated in $2 \%$ of the peptides and is not located within the predicted 14-3-3 binding motif. These data support the Phos-tag analysis (Fig. 6A), showing that XopO is not commonly phosphorylated in planta under these conditions. It is likely that XopO binds to TFT at either a nonconserved sequence or at a 14-3-3 binding site that is not phosphorylated.

For XopE2, peptide coverage was $87.9 \%$ indicating that some phosphorylated residues may have been missed. Five residues were phosphorylated in XopE2 (Fig. 6B). One of these, T66, is located in a predicted 14-3-3 binding site. In all,

A

\begin{tabular}{|c|c|c|c|c|c|c|c|c|c|}
\hline & \multicolumn{4}{|c|}{ Input } & \multicolumn{5}{|c|}{ Pull-down } \\
\hline 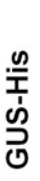 & 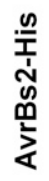 & 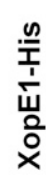 & 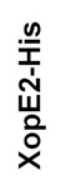 & 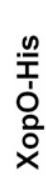 & 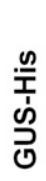 & 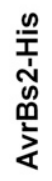 & 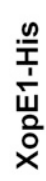 & 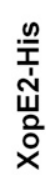 & $\begin{array}{l}\text { 몸 } \\
\text { 음 } \\
\text { 음 }\end{array}$ \\
\hline 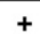 & - & + & + & + & + & - & + & + & + \\
\hline - & + & - & - & - & - & + & - & - & - \\
\hline
\end{tabular}
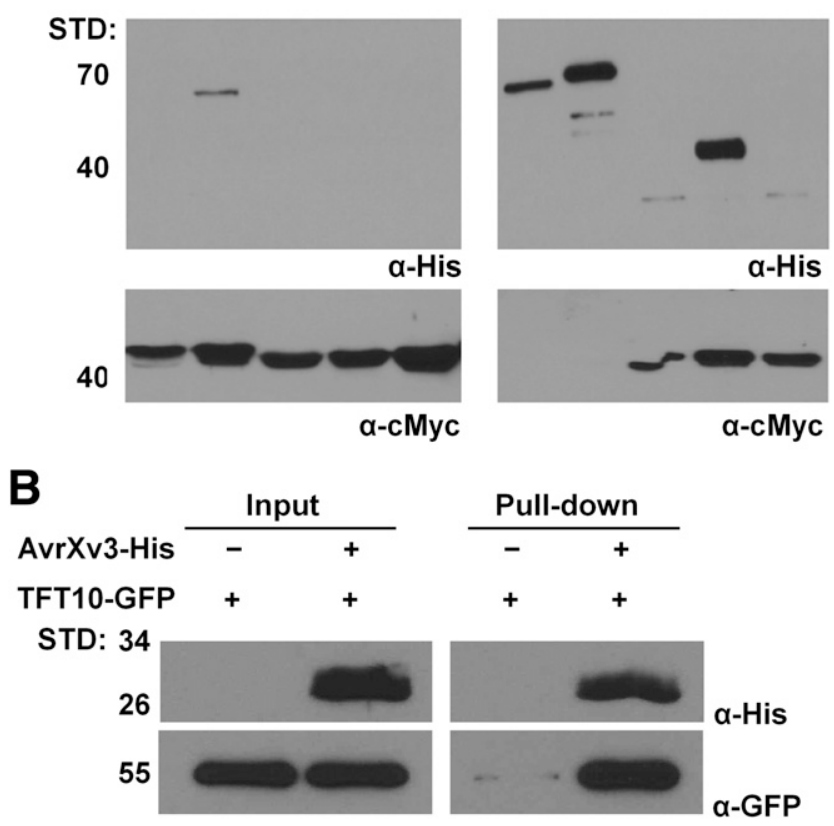

Fig. 4. Affinity purification of XopE1, XopE2, XopO, and AvrXv3 with tomato 14-3-3 isoform (TFT)10 in planta. Nicotiana benthamiana leaves were inoculated with a suspension $\left(0.8 \times 10^{9} \mathrm{CFU} / \mathrm{ml}\right)$ of Agrobacterium tumefaciens strains expressing GUS-His or effector-His and TFT-cMycVENUS $^{\mathrm{N}}$ isoforms (TFT10-V or TFT5-V) or TFT10-GFP. After $48 \mathrm{~h}$, proteins were purified by $\mathrm{Ni}^{+}$affinity chromatography and analyzed by immunoblot analysis using anti-His and anti-cMyc sera (detects VENUS ${ }^{\mathrm{N}}$ with $\mathrm{cMyc}$ tag). XopE1-His and XopO-His were detected at expected molecular mass (MW) after overnight exposure. The experiment was repeated at least three times. Symbols: $+=$ protein expressed and $-=$ vector control. MW standard $(\mathrm{STD})=\mathrm{kDa}$. A, Expected protein MW: GUS-His = $71 \mathrm{kDa}$, AvrBs2-His $=81 \mathrm{kDa}$, XopE1-His = $46 \mathrm{kDa}$, XopE2-His $=41 \mathrm{kDa}$, XopO-His $=26 \mathrm{kDa}$, TFT5-cMyc-VENUS ${ }^{\mathrm{N}}=51 \mathrm{kDa}$, and TFT10-cMycVENUS $^{\mathrm{N}}=51 \mathrm{kDa}$. B, Expected protein MW: AvrXv3-His $=26 \mathrm{kDa}$ and TFT10-GFP $=57.7 \mathrm{kDa}$.
$39 \%$ of T66 residues were phosphorylated, suggesting that it may be a 14-3-3 phospho-binding site.

\section{XopE2 binding to TFT10 is dependent on mutant T66/T131/T334.}

Based on the MS phosphorylation data, two XopE2 phosphorylated residues (T66 and S334) were mutagenized via sitedirected mutagenesis to determine whether they are required for binding to TFT. T66 and S334 were substituted with alanine to prevent phosphorylation. T66 was selected because it is located within a putative 14-3-3 binding motif. S334 was selected because it was highly phosphorylated ( $81 \%$ of peptides) (Fig. 6B). A XopE2 double mutant (T66A/S334) was then used in BiFC assays with TFT10, using glutathione- $S$-transferase (GST) as a negative control (Taylor et al. 2012).

Fluorescence was detected near the plasma membrane when XopE2(T66A/S334) was coexpressed with TFT10, although fluorescence was weaker than that observed for XopE2 and TFT10 (Supplementary Fig. S8). This suggested that the binding of the XopE2 mutants to TFT10 and TFT5 was reduced but not abolished. BiFC analysis was repeated with a triple mutant, XopE2(T66A/T131A/T334A), to determine whether binding could be further disrupted. Alanine substitution for T131 was chosen because T131 is the only other residue predicted to be in a 14-3-3 binding site. Compared with wildtype XopE2, less fluorescence was observed when the XopE2 (T66A/T131A/T334A) triple mutant was coexpressed with TFT10 or TFT5 (Fig. 7 E, F, H, and I), despite stable expression of the triple mutant (Supplementary Fig. S5). These data suggest that $\mathrm{T} 131$ or all three residues are required for maximal binding of XopE2 to TFT10 and TFT5. Notably, the abundance of TFT10 and TFT5 was less when each was coexpressed with XopE2(T66A/T131A/T334A) compared with levels detected for coexpression with GST or wild-type XopE2. One interpretation of these findings is that XopE2 binding to TFT10 and TFT5 increases their stability in planta. Similar findings were reported for the detection of a stable TFT1/XopN/TARK1 complex (Taylor et al. 2012). The detection of fluorescence for

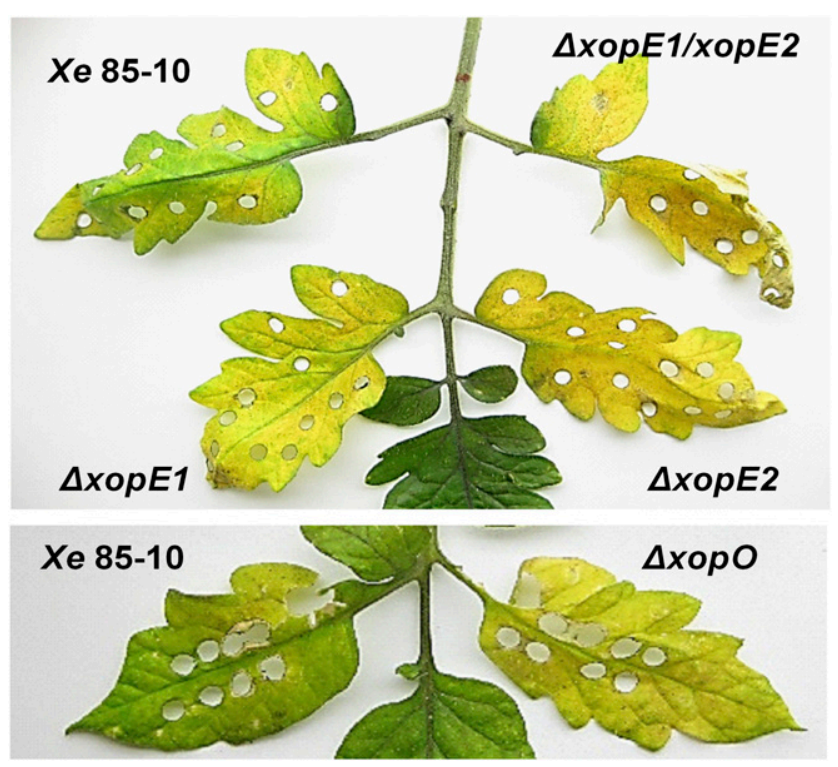

Fig. 5. Phenotype of Xanthomonas euvesicatoria (Xe) 85-10 mutant strains in infected tomato leaves. Susceptible VF36 tomato leaves were inoculated with bacteria at $1 \times 10^{5} \mathrm{CFU} / \mathrm{ml}$. Symptom development was photographed 9 days postinoculation. Experiment was performed at least three times. Top panel: X. euvesicatoria 85-10, $\triangle$ xopE1, $\triangle x o p E 2$, and $\triangle$ xopE1xopE2. Bottom panel: $X$. euvesicatoria $85-10$ and $\triangle$ xopO. 
the coexpression of the triple mutant with TFT10 or TFT5 in the BiFC assay suggests that there are likely other residues in XopE2 that facilitate binding to these TFT or that phosphorylation of the residues is not required.

Finally, we determined whether the residues in XopE2 that contribute TFT binding (Fig. 7) are required for the suppression of symptom development in X. euvesicatoria-infected leaves. We quantified the level of tissue chlorosis by measuring the chlorophyll content in $X$. euvesicatoria-infected leaves. Leaves infected with wild-type $X$. euvesicatoria, the $\Delta x o p E 2$ mutant, or the $\triangle x o p E 2$ mutant complemented with either wild-type $\triangle x o p E 2$ or the triple mutant $\Delta x o p E 2(T 66 A / T 131 A / S 334 A)$ grew to similar titers at 10 dpi (Supplementary Fig. S9). However, tomato leaves infected with wild-type $X$. euvesicatoria had higher chlorophyll content compared with leaves infected with the $\triangle x o p E 2$ mutant. This was also observed for the $\triangle x o p E 2$ mutants complemented with wild-type $x o p E 2$ or the xopE2 triple mutant. These data indicate that XopE2 residues T66, T133, and S334, involved in binding to TFT proteins, are not required to suppress chlorosis or promote bacterial growth in tomato leaves.

\section{DISCUSSION}

The existence of large 14-3-3 families in plants raises important issues regarding isoform redundancy and functional diversity (Paul et al. 2012). Our survey of TFT functions during ETI suggests that specialization evolved in response to biotic stress. Of the 11 14-3-3 isoforms, we found that 6 contribute to $X v 3$-mediated disease resistance (i.e., TFT1, TFT4, TFT5, TFT6, TFT10, and TFT11) (Fig. 2). In terms of relatedness, TFT proteins group into pairs; TFT1 with TFT10, TFT4 with TFT11, and TFT5 with TFT6 (Lozano-Durán and Robatzek 2015). Whether these isoforms are functionally redundant requires the generation of multiple TFT gene mutants, as well as examination of isoform spatial and temporal expression.

Notably, there was congruence of our TFT mRNA expression data during $X$. euvesicatoria-elicited ETI with that observed during $P$. syringae-elicited ETI (Pombo et al. 2014). Significant induction of four TFT isoforms (TFT1, TFT5, TFT10, and TFT11) by 6 hpi was detected in Pto-resistant tomato leaves. By contrast, a different group of mRNA (TFT3, TFT5, and TFT7) was induced during Pseudomonas-elicited PTI (Rosli et al. 2013).

The identity and diversity of 14-3-3 complexes formed during plant-pathogen interactions is largely unknown. However, several protein-protein interaction screens indicate that T3SE associated with 14-3-3 proteins and formation of effector-14-33 complexes impact the outcome of host infections (LozanoDurán and Robatzek 2015). For example in Xanthomonas-host interactions, the XopN effector promotes virulence in tomato leaves by attenuating PTI signaling at early stages of infection (Kim et al. 2009; Taylor et al. 2012), whereas XopQ promotes virulence in pepper by interfering with ETI signaling (Teper et al. 2014). Both effectors appear to directly hinder the function of 14-3-3 isoforms operating as positive regulators of immune signaling. Although the mechanisms of suppression are not known, it is proposed that XopN stabilizes TFT1 in an inactive PTI signaling complex with the pseudokinase TARK1 at the plant plasma membrane (Taylor et al. 2012) and XopQ competes with TFT4 client proteins for binding to TFT4 during ETI (Teper et al. 2014). By contrast, another effector-14-3-3 association is linked to activation of immune signaling. The Xanthomonas AvrRxv effector binds TFT9 and mutations that disrupt this interaction interfere with the activation of AvrRxv-dependent ETI in tomato (Whalen et al. 2008). Whether the activity of T3SE of plant pathogens requires association with host 14-3-3 proteins remains an open question.

All of the X. euvesicatoria T3SE examined had predicted 143-3 binding sites; however, our targeted interaction screen in yeast using 11 TFT revealed that only a subset of the effectors associate with 14-3-3 proteins. Two known 14-3-3-interacting proteins, XopN and XopQ, were identified (Kim et al. 2009; Taylor et al. 2012; Teper et al. 2014). Five novel effectors (XopE1, XopE2, XopO, XopAU, and AvrXv3) were found to physically interact with multiple TFT proteins in yeast and selected isoforms in planta (Figs. 3, 4, and 7). It is important to note that these interactions were captured using two heterologous systems (i.e., yeast and $N$. benthamiana). It is possible that additional $X$. euvesicatoria effector-14-3-3 complexes may form in host tissues during natural infection.

Intriguingly, we found that multiple 14-3-3 proteins are required for $X v 3$ resistance (Fig. 2) and that AvrXv3 interacts with TFT10 in planta (Fig. 4B). We now hypothesize that an immune complex comprising one or more 14-3-3 proteins functions in the initiation or signaling for $X v 3$-dependent ETI in tomato. Given that XopQ suppresses ETI in pepper (Teper et al. 2014), genetic analyses were performed to determine whether any of the identified effectors are required to suppress or enhance AvrXv3-dependent ETI in Xv3 tomato leaves. We did not detect any differences in electrolyte leakage that corresponded with a significant change in bacterial titer in $X v 3$ tomato leaves infected with $X$. euvesicatoria effector null mutants expressing AvrXv3 when compared with wild-type $X$. euvesicatoria expressing AvrXv3. These data suggest that the identified $X$. euvesicatoria effectors are interacting with distinct 14-3-3 complexes in planta.

Genetic analyses for XopE1, XopE2, and XopO were performed to determine whether one or more of these effectors is
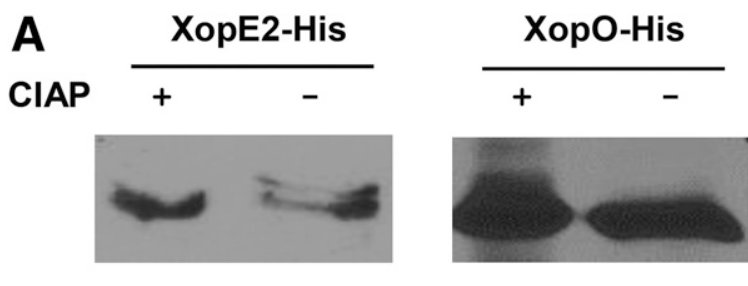

B

\begin{tabular}{|l|c|l|l|}
\hline Effector & Position & Residue & $\%$ Phosphorylated \\
\hline XopE2 & 43 & Ser & $100 \%$ (1 peptide) \\
\hline & 66 & Thr & $39 \%$ \\
\hline & 224 & Ser & $100 \%$ (1 peptide) \\
\hline & 309 & Ser & $45 \%$ \\
\hline & 334 & Ser & $81 \%$ \\
\hline XopO & 47 & Ser & $2 \%$ \\
\hline
\end{tabular}

Fig. 6. Phosphorylation state of XopE2 and XopO in plant extracts. A, Phos-tag gel analysis of XopE2-His and XopO-His. Proteins were transiently expressed in Nicotiana benthamiana leaves, affinity purified, treated with (+) or without (-) calf intestinal alkaline phosphatase (CIAP) for 15 min at $37^{\circ} \mathrm{C}$, separated in sodium dodecyl sulfate polyacrylamide gel

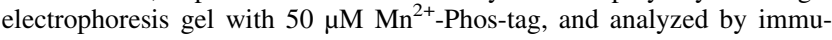
noblot analysis using anti-His sera. B, Summary of phosphorylated residues in purified XopE2 and XopO determined by liquid chromatography-mass spectrometry. 


\section{A GST-SCFP3AC + GST-VENUS ${ }^{\mathrm{N}}$}

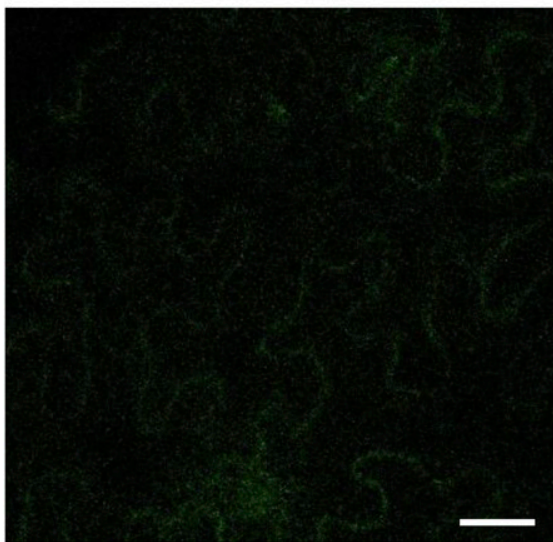

D XopE2-SCFP3AC + GST-VENUS ${ }^{N}$

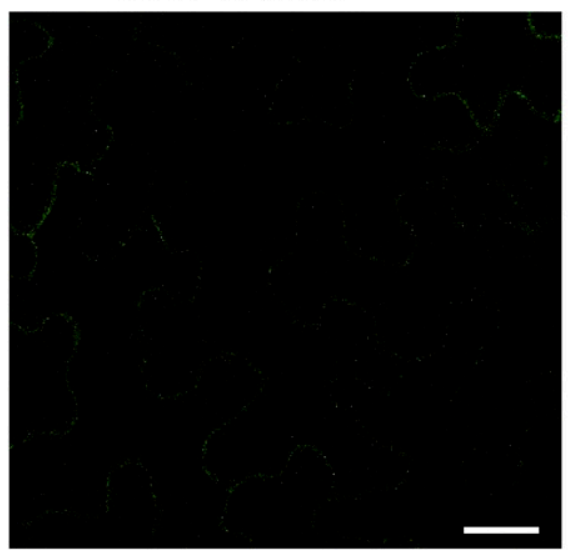

\section{G T66A/T131A/S334A- SCFP3AC + GST-VENUS ${ }^{N}$}

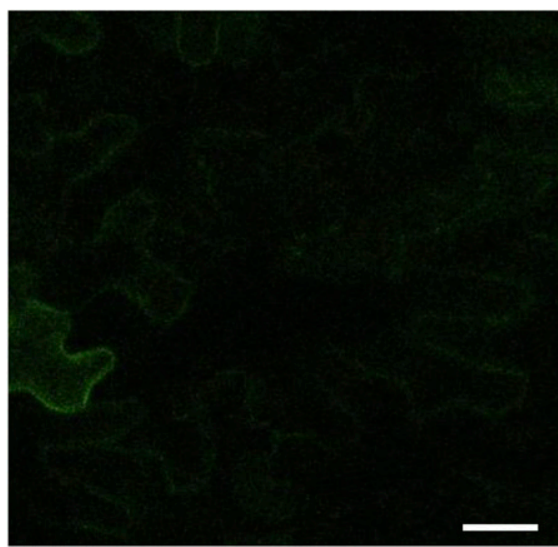

B GST-SCFP3AC + TFT5-VENUS ${ }^{\mathrm{N}}$

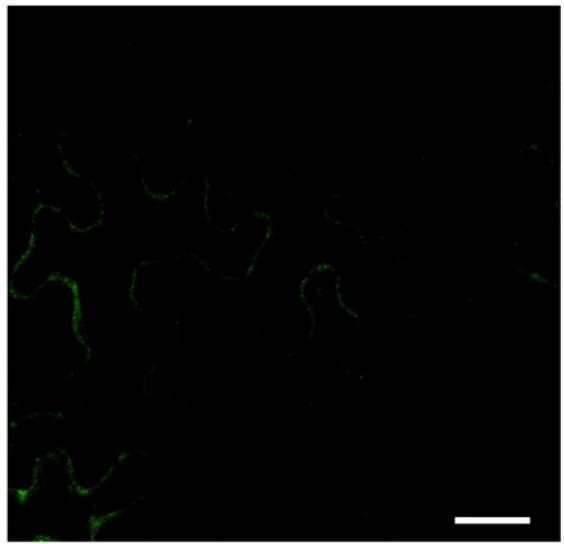

E XopE2-SCFP3AC + TFT5-VENUS ${ }^{\mathrm{N}}$

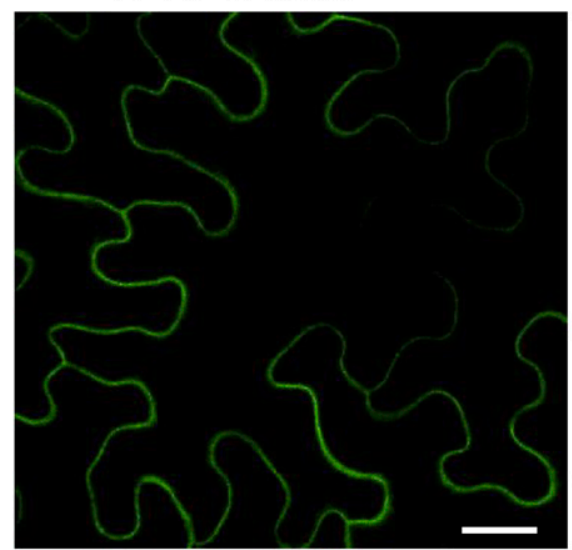

H T66A/T131A/S334ASCFP3A ${ }^{\mathrm{C}}+$ TFT5-VENUS $^{\mathrm{N}}$

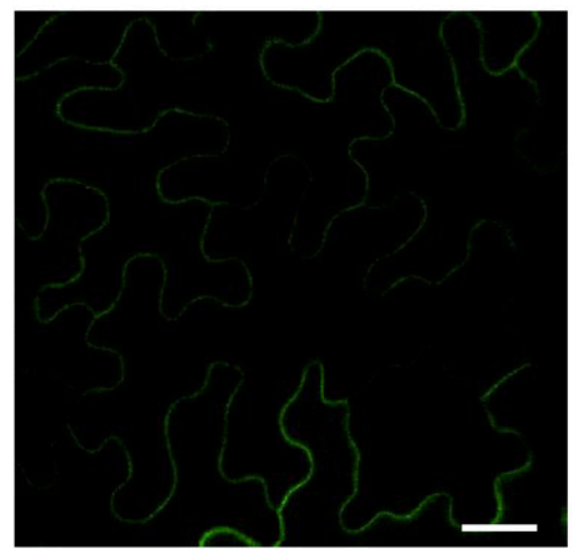

C GST-SCFP3AC + TFT10-VENUS ${ }^{\mathrm{N}}$

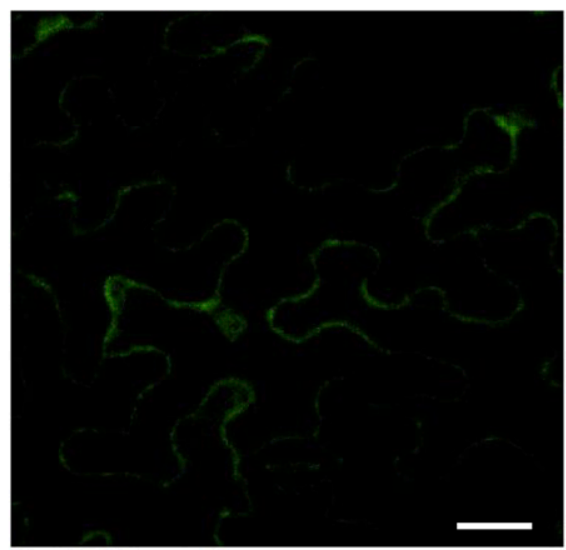

F XopE2-SCFP3AC + TFT10-VENUS ${ }^{\mathrm{N}}$

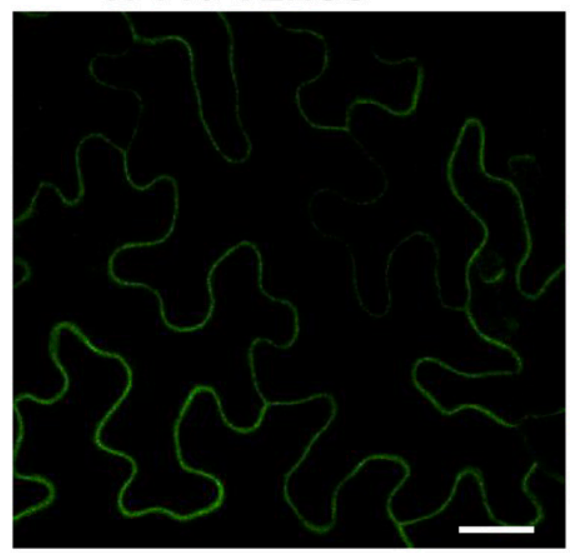

I T66A/T131A/S334ASCFP3AC + TFT10-VENUS ${ }^{N}$

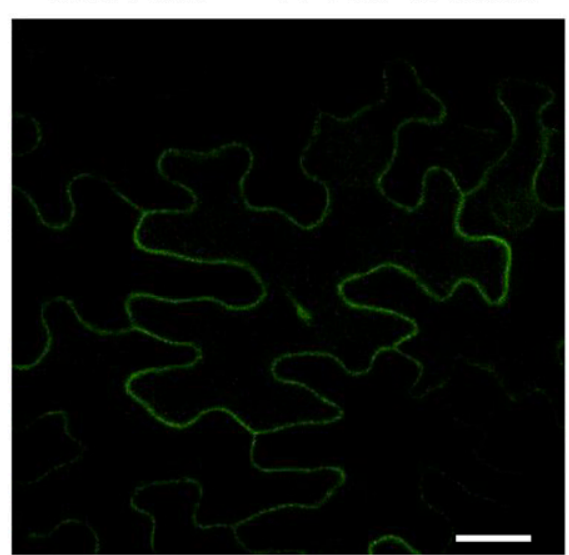

Fig. 7. Bimolecular fluorescence complementation analysis of wild-type XopE2 and mutant XopE2(T66A/T131A/S334A) with tomato 14-3-3 isoform (TFT) 5 and TFT10 in planta. Nicotiana benthamiana leaves were hand-infiltrated with a suspension $\left(0.8 \times 10^{9} \mathrm{CFU} / \mathrm{ml}\right.$ concentration) of two Agrobacterium tumefaciens strains expressing different fusion proteins. Leaf epidermal cells were visualized $62 \mathrm{~h}$ postinoculation by confocal microscopy at $\times 60$. Bar $=20 \mu \mathrm{m}$. $\mathbf{A}, \mathbf{B}, \mathbf{C}, \mathbf{D}$, and $\mathbf{G}$, GST protein fusions (GST-SCFP3A ${ }^{\mathrm{C}}$ and GST-VENUS ${ }^{\mathrm{N}}$ ) were used as negative controls to monitor background fluorescence. E, F, Wildtype (XopE2-SCRP3A ${ }^{\mathrm{C}}$ ) or $\mathbf{H}$ and I, mutant XopE2 (XopE2(T66A/T131A/S334A)-SCRP3A ${ }^{\mathrm{C}}$ ) was coexpressed with TFT5-VENUS ${ }^{\mathrm{N}}$ or TFT10-VENUS ${ }^{\mathrm{N}}$. These data are representative of results obtained in two independent experiments. 
required for $X$. euvesicatoria pathogenesis in susceptible tomato plants. X. euvesicatoria null mutants lacking xopE1, xopE2, xорE1/xopE2, or $x o p O$ grew to titers equivalent to those of wild-type $X$. euvesicatoria in VF36 tomato leaves at $10 \mathrm{dpi}$, indicating that these effectors are not required for bacterial multiplication in planta. However, XopE1, XopE2, and XopO are required to suppress chlorosis and tissue collapse at very late stages of Xanthomonas infection (Fig. 5). This phenotype appeared to be additive because the xopE1/xopE2 double mutant developed symptoms earlier than those observed for the single deletion mutants (Fig. 5). These results suggest that XopE1, XopE2, and XopO may function redundantly to inhibit $X$. euvesicatoria-induced chlorosis in tomato leaves.

Phosphorylation of 14-3-3 clients is often a determinant for 14-3-3 binding. Many T3SE are predicted to have 14-3-3 binding motifs (Giska et al. 2013), suggesting that phosphorylation of effectors inside plant cells may be necessary for them to associate with 14-3-3 proteins. This was shown to be the case for the T3SE XopN, XopQ, and HopQ (Giska et al. 2013; Kim et al. 2009; Taylor et al. 2012; Teper et al. 2014) but not for ExoS from $P$. aeruginosa (Henriksson et al. 2002).

Our phospho-peptide analyses revealed that XopE2 but not XopO was phosphorylated in $N$. benthamiana leaves (Fig. 6B). Five phosphorylation sites were mapped in the XopE2 protein. Of these, three residues (T66, T131, and S334) are required for maximal binding to TFT10 (Fig. 7), indicating that phosphorylation facilitates the association of XopE2 with TFT10. However, these residues were not required to suppress leaf chlorosis in $X$. euvesicatoria-infected tomato leaves. It is possible that the phosphorylation state of XopE2 is different during X. euvesicatoria infection in tomato or that XopE2-14-3-3 complexes in tomato are not involved in signaling pathways controlling leaf symptom development. Although we weren't able to confirm that the interaction between XopE2 and 14-3-3 proteins plays a role in the chlorosis phenotype, further investigation is warranted because leaf chlorosis is linked to the production of the defense hormone ethylene (Lund et al. 1998) and 14-3-3 proteins are important regulators of the rate limiting enzyme (ACS1) in the ethylene biosynthesis pathway (Yoon and Kieber 2013).

In conclusion, we show that several TFT isoforms contribute to $X v 3$ disease resistance in tomato in response to $X$. euvesicatoria infection. We also report that a subset of $X$. euvesicatoria T3SE interact with TFTs in planta, further highlighting the importance of 14-3-3 proteins in host-pathogen interactions. The complexity on both sides of this interaction (multiple TFT isoforms and $X$. euvesicatoria T3SE) necessitates the isolation of individual effector-14-3-3 complexes to gain further insight into the function and specificity of 14-3-3 proteins in immune signaling or disease symptom development.

\section{MATERIALS AND METHODS}

\section{Bacterial strains.}

Strains used in this study were as follows: Escherichia coli DH5 $\alpha$; Agrobacterium tumefaciens C58C1 (pCH32) and GV3101; X. euvesicatoria strains 5746, 5746 pDSK519 $(a v r X v 3)$, and 85-10 (wild type, $\Delta h r c V, \Delta x o p E 1, \Delta x o p E 2$, $\Delta x o p E 1 / \Delta x o p E 2, \Delta x o p N, \Delta x o p O, \Delta x o p P, \Delta x o p Q$, and $\Delta x o p A U)$. $E$. coli and $A$. tumefaciens were grown on Luria-Bertani agar medium at 37 and $28^{\circ} \mathrm{C}$, respectively. X. euvesicatoria strains were grown on nutrient yeast glycerol agar (Turner et al. 1984) at $28^{\circ} \mathrm{C}$.

\section{$X$. euvesicatoria effector mutant strains.}

$X$. euvesicatoria strain 85-10 effector null mutants were constructed using homologous recombination, as described (Kim et al. 2008), then analyzed by PCR to confirm that deletion occurred at each effector locus. To construct an $X$. euvesicatoria 85-10 $\Delta x o p E 1 \Delta x o p E 1, \Delta x o p E 2$, and $\triangle x o p E 1 \Delta$ xopE2 deletion mutant, pK18E1 and pK18E2 plasmids were introduced into $X$. euvesicatoria $85-10$ by triparental mating. Then, the homologous recombination was carried out as described by Thieme et al. (2007). Plasmids containing wild-type or mutant $x o p E 2$ expressed from the xopE2 promoter were mated into the $X$. euvesicatoria $\triangle$ xopE2 mutant for complementation assays.

\section{Plant lines.}

Solanum lycopersicum cultivar VF36 (susceptible to $X$. euvesicatoria 85-10 and 5746) and Hawaii $7981 X v 3$ plants (resistant to $X$. euvesicatoria 5746 expressing avrXv3) were used for bacterial growth curve assays. $N$. benthamiana was used for transient protein expression assays.

\section{Plasmid constructions.}

PCR was used to engineer restriction sites to construct gene fusions. Primer sequences used for PCR are listed in Supplementary Table S2. PCR-generated DNA fragments were cloned into pCR8/GW/TOPO and Gateway technology (Invitrogen) was used to recombine genes into vectors for expression in $X$. euvesicatoria, yeast, and plants. Plasmids used are listed in Supplementary Table S3.

\section{qPCR.}

qPCR was performed using cDNA isolated from tomato leaves infiltrated with $10 \mathrm{mM} \mathrm{MgCl}{ }_{2}$ (mock), X. euvesicatoria strain 5746 (compatible interaction) or $X$. euvesicatoria strain 5746 expressing $a v r X v 3$ (incompatible interaction) with genespecific primers and SYBR Premix Ex Taq II (Tli RNase H Plus) (Takara), as described (Teper et al. 2014). Reactions were carried out with an Mx3000P qPCR system (Stratagene). GAPDH expression was used to normalize the TFT isoform mRNA level in each sample. Relative expression values were determined using the comparative cycle threshold method $\left(2^{-\Delta \Delta \mathrm{Ct}}\right)$ (Pfaffl 2001)

\section{VIGS.}

DNA fragments (200- to 250-bp fragment of $3^{\prime}$ untranslated region [UTR] of TFT genes) engineered to target the TFT isoform genes TFT1, TFT4, TFT5, TFT6, TFT10, and TFT11 were recombined into binary plasmid pTRV2. We chose the $3^{\prime}$ UTR of each gene in attempt to prevent silencing of related TFT family members. Binary plasmids were transformed into A. tumefaciens C58 (pGV2260) and used for VIGS, as described (Melech-Bonfil and Sessa 2010). Silenced Hawaii 7981 tomato lines were analyzed by qPCR and bacterial growth curve analysis to monitor TFT isoform silencing and $X$. euvesicatoria growth, respectively.

\section{Bacterial growth curves.}

To measure $X$. euvesicatoria growth in planta, tomato leaves were vacuum infiltrated or hand inoculated with a suspension $\left(1 \times 10^{5}\right.$ or $\left.1 \times 10^{8} \mathrm{CFU} / \mathrm{ml}\right)$ of bacteria in $10 \mathrm{mM} \mathrm{MgCl}$. Plants were kept under $16 \mathrm{~h}$ light/day at $28^{\circ} \mathrm{C}$. Three $1-\mathrm{cm}^{2}$ leaf disks were sampled from five independent plants and ground in $1 \mathrm{ml}$ of $10 \mathrm{mM} \mathrm{MgCl}_{2}$ for determining bacterial titer at 0,4 , and 8 dpi. Leaf symptom development was monitored until $12 \mathrm{dpi}$. The experiment was repeated three times with similar results.

\section{Electrolyte leakage assay.}

Three leaf discs $(1.5 \mathrm{~cm}$ in diameter $)$ were sampled from infiltrated areas of at least seven plants, and floated in $5 \mathrm{ml}$ water for $4 \mathrm{~h}$ at $25^{\circ} \mathrm{C}$ with shaking. Conductivity was measured using a DD2-12DW conductivity meter (Bante Instruments). The experiment was repeated three times with similar results. 


\section{Yeast constructs and interaction screen.}

Effector genes $(n=37)$ from $X$. euvesicatoria strain $85-10$ were cloned into $\mathrm{pCR} 8 / \mathrm{GW} / \mathrm{TOP}$ and then recombined into the LexA bait plasmid pEG202nls to create 37 pEG202nls(effector) plasmids encoding for LexA-NLS-effector fusion proteins. TFT isoform CDNA (TFT1 to TFT11) were cloned into the prey plasmid pJG4-5 to create 11 pJG4-5(TFT) plasmids encoding for AD-TFT fusion proteins. Plasmids were transformed into yeast strain EGY48 pSH18-34 and screened as described (Kim et al. 2009). Protein expression was determined by immunoblot analysis.

\section{Immunoblot analysis.}

Proteins were separated by SDS-PAGE, transferred to nitrocellulose, then detected by ECL prime (GE) using anti-HA (Covance), anti-cMyc (Covance), or anti-6xHis (Qiagen) and horseradish peroxidase-conjugated secondary antibodies (BioRad). Primary and secondary antibodies were typically diluted $1: 3000$ to $1: 5000$ and $1: 3000$, respectively.

\section{Transient protein expression in $N$. benthamiana.}

A. tumefaciens strains grown overnight at $28^{\circ} \mathrm{C}$ were collected and incubated in induction media $(10 \mathrm{mM} 2-(N$-morpholino) ethanesulfonic acid [pH 5.6], $10 \mathrm{mM} \mathrm{MgCl} 2$, and $150 \mu \mathrm{M}$ acetosyringone) for $2 \mathrm{~h}$. Leaves were inoculated with a final suspension $\left(0.8 \times 10^{9} \mathrm{CFU} / \mathrm{ml}\right)$ of two strains. Plants were incubated at ambient temperature under continuous low light for 48 to $72 \mathrm{~h}$.

\section{BiFC.}

Effector and TFT cDNAs were recombined into BiFC destination vectors (Gehl et al. 2009) $\mathrm{pDEST}^{-{ }^{\mathrm{GW}} \mathrm{SCYCE} \text { or }}$ pDEST- ${ }^{\mathrm{GW}}$ VYNE to generate fusion proteins with HA-SCFP3A ${ }^{\mathrm{C}}$ or cMyc-VENUS ${ }^{\mathrm{N}}$, respectively. The resulting $\mathrm{BiFC}$ vectors were transformed into A. tumefaciens $\mathrm{C} 58 \mathrm{C} 1 \mathrm{pCH} 32$ and then transiently expressed in $N$. benthamiana leaves. Fluorescence was visualized 48 hpi using a Leica TCS SP5 confocal microscope with Leica LAS AF software. Proteins were excited with an argon laser $(488 \mathrm{~nm})$ and emitted light was captured at $510 \mathrm{~nm}$.

\section{Affinity purification assay.}

$N$. benthamiana leaves were infiltrated with two A. tumefaciens strains: one expressing a His-tagged effector or control (AvrBs2-His, XopE1-His, XopE2-His, XopO-His, or GUS-His) and a cMyc-VENUS ${ }^{\mathrm{N}}$-tagged TFT isoform (TFT5VENUS $^{\mathrm{N}}$ or TFT10-VENUS ${ }^{\mathrm{N}}$ ). At 48 to $72 \mathrm{hpi}$, approximately $3 \mathrm{~g}$ of tissue was frozen in liquid nitrogen, ground, solubilized in $15 \mathrm{ml}$ of extraction buffer $\left(100 \mathrm{mM} \mathrm{NaH} \mathrm{PO}_{4}[\mathrm{pH} 8]\right.$, $150 \mathrm{mM} \mathrm{NaCl}, 10 \mathrm{mM}$ imidazole, protease inhibitor cocktail [Sigma], $2 \mathrm{mM} \mathrm{Na}_{3} \mathrm{VO}_{4}, 5 \mathrm{mM} \mathrm{NaF}$, and $0.25 \%$ Triton X-100) and centrifuged for $20 \mathrm{~min}$ at $10,000 \times g$ at $4^{\circ} \mathrm{C}$. Supernatants were filtered through Mira-cloth (Calbiochem). Soluble extracts were incubated with $100 \mu \mathrm{l}$ of a $50 \%$ slurry of $\mathrm{Ni}$ nitrilotriacetic acid Superflow agarose (Qiagen) at $4^{\circ} \mathrm{C}$ for $1 \mathrm{~h}$. Agarose was recovered by centrifugation $(425 \times g)$ at $4^{\circ} \mathrm{C}$ and washed three times with extraction buffer. Proteins were eluted with $60 \mu \mathrm{l}$ of Laemmli buffer (65.8 mM Tris-HCl [pH 6.8], 2.1\% SDS, $26.3 \%$ glycerol, and $0.01 \%$ bromophenol blue) and analyzed by immunoblot analysis.

\section{Phos-tag gel analysis.}

Phosphate affinity SDS-PAGE using acrylamide-pendant Phos-tag (Wako Pure Chemicals) was performed using affinitypurified XopE2-His and XopO-His. Proteins were incubated with buffer (50 mM Tris- $\mathrm{HCl}$ [pH 7.9], $100 \mathrm{mM} \mathrm{NaCl}, 10 \mathrm{mM}$ $\mathrm{MgCl}_{2}$, and $1 \mathrm{mM}$ dithiothreitol) and $20 \mathrm{U}$ of CIAP (Sigma) or water at $37^{\circ} \mathrm{C}$ for $15 \mathrm{~min}$. Proteins were separated by $10 \%$ SDS-
PAGE with $50 \mu \mathrm{M} \mathrm{Mn}{ }^{2+}$-Phos-tag, then analyzed by immunoblot blot analysis using anti-His sera.

\section{MS.}

XopE2-His and XopO-His were purified from $15 \mathrm{~g}$ of tissue as described above using denaturing buffer (100 mM sodium phosphate and $10 \mathrm{mM}$ Tris [pH 8], $8 \mathrm{M}$ urea, $1 \%$ CHAPS detergent, $5 \mathrm{mM} \mathrm{NaF}, 2 \mathrm{mM} \mathrm{Na}_{3} \mathrm{VO}_{4}$, and protease inhibitor cocktail [Sigma]). Proteins were excised from SDS-PAGE gels stained with Coomassie Brilliant Blue and digested with trypsin. Phosphopeptides were enriched using $\mathrm{TiO}_{2}$ and high-performance liquid chromatography and analyzed by MS at Stanford Mass Spectrometry Facility, as described (Taylor et al. 2012).

\section{Site-directed mutagenesis.}

XopE2 mutants (alanine substitutions at T66, T131, and S334) were generated with a QuikChange site-directed mutagenesis kit (Stratagene) using pCR8/GW/TOPO(xopE2) as template and primers. Mutations were confirmed by DNA sequence analysis.

\section{Chlorophyll quantification.}

Total chlorophyll in leaves was determined as described (Arnon 1949), with slight modification. VF36 tomato leaves were hand inoculated with a needleless syringe with a $1 \times 10^{5}$ $\mathrm{CFU} / \mathrm{ml}$ suspension of $X$. euvesicatoria in $10 \mathrm{mM} \mathrm{MgCl}_{2}$. At 10 dpi, four leaf discs $\left(1 \mathrm{~cm}^{2}\right)$ from six biological replicates were pooled, ground in $1 \mathrm{ml}$ of $80 \%$ acetone, then centrifuged at $18,000 \times g$ for $1 \mathrm{~min}$. Optical density of the supernatant at 645 and $663 \mathrm{~nm}$ was determined to calculate total chlorophyll content. The experiment was repeated three times, and the average chlorophyll content \pm standard deviation is reported.

\section{ACKNOWLEDGMENTS}

We thank members of the Sessa and Mudgett laboratories for critical discussion.

\section{LITERATURE CITED}

Arnon, D. I. 1949. Copper enzymes in isolated chloroplasts. Polyphenoloxidase in Beta vulgaris. Plant Physiol. 24:1-15.

Astua-Monge, G., Minsavage, G. V., Stall, R. E., Davis, M. J., Bonas, U., and Jones, J. B. 2000. Resistance of tomato and pepper to T3 strains of Xanthomonas campestris pv. vesicatoria is specified by a plant-inducible avirulence gene. Mol. Plant-Microbe Interact. 13:911-921.

Balaji, V., Gibly, A., Debbie, P., and Sessa, G. 2007. Transcriptional analysis of the tomato resistance response triggered by recognition of the Xanthomonas type III effector AvrXv3. Funct. Integr. Genomics 7:305-316.

Bigeard, J., Colcombet, J., and Hirt, H. 2015. Signaling mechanisms in pattern-triggered immunity (PTI). Mol. Plant 8:521-539.

Borch, J., Bych, K., Roepstorff, P., Palmgren, M. G., and Fuglsang, A. T. 2002. Phosphorylation-independent interaction between 14-3-3 protein and the plant plasma membrane H+-ATPase. Biochem. Soc. Trans. 30: 411-415.

Bridges, D., and Moorhead, G. B. 2005. 14-3-3 Proteins: A number of functions for a numbered protein. Sci. STKE 2005:re10.

Camoni, L., Harper, J. F., and Palmgren, M. G. 1998. 14-3-3 Proteins activate a plant calcium-dependent protein kinase (CDPK). FEBS Lett. 430:381-384

Choi, H. W., and Klessig, D. F. 2016. DAMPs, MAMPs, and NAMPs in plant innate immunity. BMC Plant Biol. 16:232.

Coblitz, B., Shikano, S., Wu, M., Gabelli, S. B., Cockrell, L. M., Spieker, M., Hanyu, Y., Fu, H., Amzel, L. M., and Li, M. 2005. C-terminal recognition by 14-3-3 proteins for surface expression of membrane receptors. J. Biol. Chem. 280:36263-36272.

Cui, H., Tsuda, K., and Parker, J. E. 2015. Effector-triggered immunity: From pathogen perception to robust defense. Annu. Rev. Plant Biol. 66: 487-511.

Fu, H., Subramanian, R. R., and Masters, S. C. 2000. 14-3-3 Proteins: Structure, function, and regulation. Annu. Rev. Pharmacol. Toxicol. 40: 617-647. 
Ganguly, S., Weller, J. L., Ho, A., Chemineau, P., Malpaux, B., and Klein, D. C. 2005. Melatonin synthesis: 14-3-3-dependent activation and inhibition of arylalkylamine $\mathrm{N}$-acetyltransferase mediated by phosphoserine-205. Proc. Natl. Acad. Sci. U.S.A. 102:1222-1227.

Gehl, C., Waadt, R., Kudla, J., Mendel, R. R., and Hansch, R. 2009. New GATEWAY vectors for high throughput analyses of protein-protein interactions by bimolecular fluorescence complementation. Mol. Plant 2: 1051-1058.

Giska, F., Lichocka, M., Piechocki, M., Dadlez, M., Schmelzer, E., Hennig, J., and Krzymowska, M. 2013. Phosphorylation of HopQ1, a type III effector from Pseudomonas syringae, creates a binding site for host 143-3 proteins. Plant Physiol. 161:2049-2061.

Henriksson, M. L., Francis, M. S., Peden, A., Aili, M., Stefansson, K., Palmer, R., Aitken, A., and Hallberg, B. 2002. A nonphosphorylated 143-3 binding motif on exoenzyme $\mathrm{S}$ that is functional in vivo. Eur. J. Biochem. 269:4921-4929.

Kim, J. G., Li, X., Roden, J. A., Taylor, K. W., Aakre, C. D., Su, B., Lalonde, S., Kirik, A., Chen, Y., Baranage, G., McLane, H., Martin, G. B., and Mudgett, M. B. 2009. Xanthomonas T3S effector XopN suppresses PAMP-triggered immunity and interacts with a tomato atypical receptor-like kinase and TFT1. Plant Cell 21:1305-1323.

Kim, J. G., Taylor, K. W., Hotson, A., Keegan, M., Schmelz, E. A., and Mudgett, M. B. 2008. XopD SUMO protease affects host transcription, promotes pathogen growth, and delays symptom development in Xanthomonas-infected tomato leaves. Plant Cell 20:1915-1929.

Li, W., Yadeta, K. A., Elmore, J. M., and Coaker, G. 2013. The Pseudomonas syringae effector HopQ1 promotes bacterial virulence and interacts with tomato $14-3-3$ proteins in a phosphorylationdependent manner. Plant Physiol. 161:2062-2074.

Lozano-Durán, R., Bourdais, G., He, S. Y., and Robatzek, S. 2014. The bacterial effector HopM1 suppresses PAMP-triggered oxidative burst and stomatal immunity. New Phytol. 202:259-269.

Lozano-Durán, R., and Robatzek, S. 2015. 14-3-3 Proteins in plantpathogen interactions. Mol. Plant-Microbe Interact. 28:511-518.

Lund, S. T., Stall, R. E., and Klee, H. J. 1998. Ethylene regulates the susceptible response to pathogen infection in tomato. Plant Cell 10: 371-382.

Madeira, F., Tinti, M., Murugesan, G., Berrett, E., Stafford, M., Toth, R., Cole, C., MacKintosh, C., and Barton, G. J. 2015. 14-3-3-Pred: Improved methods to predict 14-3-3-binding phosphopeptides. Bioinformatics 31: 2276-2283.

Maksymowych, W. P., van der Heijde, D., Allaart, C. F., Landewé, R., Boire, G., Tak, P. P., Gui, Y., Ghahary, A., Kilani, R., and Marotta, A. 2014. $14-3-3 \eta$ is a novel mediator associated with the pathogenesis of rheumatoid arthritis and joint damage. Arthritis Res. Ther. 16:R99.

Melech-Bonfil, S., and Sessa, G. 2010. Tomato MAPKKKE is a positive regulator of cell-death signaling networks associated with plant immunity. Plant J. 64:379-391.

Muslin, A. J., Tanner, J. W., Allen, P. M., and Shaw, A. S. 1996. Interaction of 14-3-3 with signaling proteins is mediated by the recognition of phosphoserine. Cell 84:889-897.

Nomura, K., Debroy, S., Lee, Y. H., Pumplin, N., Jones, J., and He, S. Y. 2006. A bacterial virulence protein suppresses host innate immunity to cause plant disease. Science 313:220-223.

Oh, C. S., and Martin, G. B. 2011. Tomato 14-3-3 protein TFT7 interacts with a MAP kinase kinase to regulate immunity-associated programmed cell death mediated by diverse disease resistance proteins. J. Biol. Chem. 286:14129-14136.

Oh, C. S., Pedley, K. F., and Martin, G. B. 2010. Tomato 14-3-3 protein 7 positively regulates immunity-associated programmed cell death by enhancing protein abundance and signaling ability of MAPKKK alpha. Plant Cell 22:260-272.

Ottmann, C., Yasmin, L., Weyand, M., Veesenmeyer, J. L., Diaz, M. H., Palmer, R. H., Francis, M. S., Hauser, A. R., Wittinghofer, A., and Hallberg, B. 2007. Phosphorylation-independent interaction between 14-33 and exoenzyme S: From structure to pathogenesis. EMBO J. 26:902-913.

Paul, A. L., Denison, F. C., Schultz, E. R., Zupanska, A. K., and Ferl, R. J. 2012. 14-3-3 Phosphoprotein interaction networks-Does isoform diversity present functional interaction specification? Front. Plant Sci. 3:190.

Petosa, C., Masters, S. C., Bankston, L. A., Pohl, J., Wang, B., Fu, H., and Liddington, R. C. 1998. 14-3-3zeta binds a phosphorylated Raf peptide and an unphosphorylated peptide via its conserved amphipathic groove. J. Biol. Chem. 273:16305-16310.

Pfaffl, M. W. 2001. A new mathematical model for relative quantification in real-time RT-PCR. Nucleic Acids Res. 29:e45.
Pombo, M. A., Zheng, Y., Fernandez-Pozo, N., Dunham, D. M., Fei, Z., and Martin, G. B. 2014. Transcriptomic analysis reveals tomato genes whose expression is induced specifically during effector-triggered immunity and identifies the Epk1 protein kinase which is required for the host response to three bacterial effector proteins. Genome Biol. 15:492.

Potnis, N., Krasileva, K., Chow, V., Almeida, N. F., Patil, P. B., Ryan, R. P., Sharlach, M., Behlau, F., Dow, J. M., Momol, M., White, F. F., Preston, J. F., Vinatzer, B. A., Koebnik, R., Setubal, J. C., Norman, D. J., Staskawicz, B. J., and Jones, J. B. 2011. Comparative genomics reveals diversity among xanthomonads infecting tomato and pepper. BMC Genomics 12:146.

Roberts, M. R., and Bowles, D. J. 1999. Fusicoccin, 14-3-3 proteins, and defense responses in tomato plants. Plant Physiol. 119:1243-1250.

Roden, J. A., Belt, B., Ross, J. B., Tachibana, T., Vargas, J., and Mudgett, M. B. 2004. A genetic screen to isolate type III effectors translocated into pepper cells during Xanthomonas infection. Proc. Natl. Acad. Sci. U.S.A. 101:16624-16629.

Rosli, H. G., Zheng, Y., Pombo, M. A., Zhong, S., Bombarely, A., Fei, Z., Collmer, A., and Martin, G. B. 2013. Transcriptomics-based screen for genes induced by flagellin and repressed by pathogen effectors identifies a cell wall-associated kinase involved in plant immunity. Genome Biol 14:R139.

Schulze, S., Kay, S., Büttner, D., Egler, M., Eschen-Lippold, L., Hause, G., Krüger, A., Lee, J., Müller, O., Scheel, D., Szczesny, R., Thieme, F., and Bonas, U. 2012. Analysis of new type III effectors from Xanthomonas uncovers XopB and XopS as suppressors of plant immunity. New Phytol. 195:894-911.

Taylor, K. W., Kim, J. G., Su, X. B., Aakre, C. D., Roden, J. A., Adams, C. M., and Mudgett, M. B. 2012. Tomato TFT1 is required for PAMPtriggered immunity and mutations that prevent T3S effector XopN from binding to TFT1 attenuate Xanthomonas virulence. PLoS Pathog. 8: e1002768.

Teper, D., Burstein, D., Salomon, D., Gershovitz, M., Pupko, T., and Sessa, G. 2016. Identification of novel Xanthomonas euvesicatoria type III effector proteins by a machine-learning approach. Mol. Plant Pathol. 17: 398-411.

Teper, D., Girija, A. M., Bosis, E., Popov, G., Savidor, A., and Sessa, G. 2018. The Xanthomonas euvesicatoria type III effector XopAU is an active protein kinase that manipulates plant MAP kinase signaling. PLoS Pathog. 14:e1006880.

Teper, D., Salomon, D., Sunitha, S., Kim, J. G., Mudgett, M. B., and Sessa, G. 2014. Xanthomonas euvesicatoria type III effector XopQ interacts with tomato and pepper 14-3-3 isoforms to suppress effector-triggered immunity. Plant J. 77:297-309.

Thieme, F., Koebnik, R., Bekel, T., Berger, C., Boch, J., Büttner, D., Caldana, C., Gaigalat, L., Goesmann, A., Kay, S., Kirchner, O. Lanz, C., Linke, B., McHardy, A. C., Meyer, F., Mittenhuber, G., Nies, D. H., Niesbach-Klösgen, U., Patschkowski, T., Rückert, C., Rupp, O., Schneiker, S., Schuster, S. C., Vorhölter, F. J., Weber, E., Pühler, A., Bonas, U., Bartels, D., and Kaiser, O. 2005. Insights into genome plasticity and pathogenicity of the plant pathogenic bacterium Xanthomonas campestris pv. vesicatoria revealed by the complete genome sequence. J. Bacteriol. 187:7254-7266.

Thieme, F., Szczesny, R., Urban, A., Kirchner, O., Hause, G., and Bonas, U. 2007. New type III effectors from Xanthomonas campestris pv. vesicatoria trigger plant reactions dependent on a conserved $\mathrm{N}$ myristoylation motif. Mol. Plant-Microbe Interact. 20:1250-1261.

Turner, P., Barber, C., and Daniels, M. 1984. Behaviour of the transposons Tn5 and Tn7 in Xanthomonas campestris pv. campestris. Mol. Gen. Genet. 195:101-107.

Whalen, M., Richter, T., Zakhareyvich, K., Yoshikawa, M., Al-Azzeh, D. Adefioye, A., Spicer, G., Mendoza, L. L., Morales, C. Q., Klassen, V., Perez-Baron, G., Toebe, C. S., Tzovolous, A., Gerstman, E., Evans, E., Thompson, C., Lopez, M., and Ronald, P. C. 2008. Identification of a host 14-3-3 Protein that Interacts with Xanthomonas effector AvrRxv. Physiol. Mol. Plant Pathol. 72:46-55.

Yaffe, M. B., Rittinger, K., Volinia, S., Caron, P. R., Aitken, A., Leffers, H., Gamblin, S. J., Smerdon, S. J., and Cantley, L. C. 1997. The structural basis for 14-3-3:phosphopeptide binding specificity. Cell 91:961-971.

Yang, X., Wang, W., Coleman, M., Orgil, U., Feng, J., Ma, X., Ferl, R., Turner, J. G., and Xiao, S. 2009. Arabidopsis 14-3-3 lambda is a positive regulator of RPW8-mediated disease resistance. Plant J. 60:539-550.

Yoon, G. M., and Kieber, J. J. 2013. 14-3-3 Regulates 1-aminocyclopropane-1carboxylate synthase protein turnover in Arabidopsis. Plant Cell 25: 1016-1028. 